\title{
A Implantação do Programa de Saúde da Família (PSF) no Estado de São Paulo (1995-2002)
}

\section{The Implementation of the Family Health Program (PSF) in the State of São Paulo (1995-2002)}

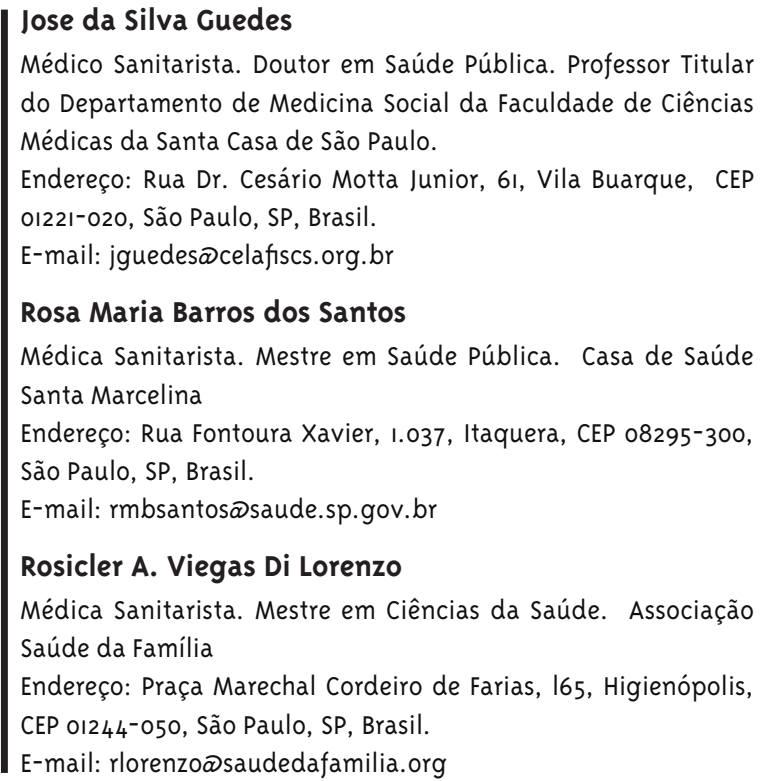

E-mail: rlorenzoळsaudedafamilia.org

\section{Resumo}

Este artigo relata a implantação do Programa de Saúde da Família no Estado de São Paulo desde seu início até o ano de 2002. São mencionados como antecedentes importantes outros programas do Ministério da Saúde, como o PIASS (1976-1979), o PACS (1991) e o Projeto do Vale do Ribeira da SESSP (1980-1987). Destaca-se também a importância do Programa metropolitano de Saúde na década de 1980 e do Movimento Popular de Saúde na Cidade de São Paulo desde a década de 1970. São descritas as principais características do modelo, a composição inicial da equipe mínima e a incorporação de novos atores no programa. São destacados três mecanismos de ação do Governo Estadual em relação ao desenvolvimento do PSF, sendo um de apoio técnico, outro de apoio técnico e financeiro e o terceiro de contratação direta de entidades filantrópicas para a implantação do programa no Município de São Paulo. A avaliação de todas as equipes de saúde da família implantadas no Estado de São Paulo, realizada em 2002, demonstrou grande aceitação da população em relação à atenção desenvolvida e identificou os problemas nos mecanismos de contratação dos profissionais da equipe. Este artigo descreve a preparação das equipes de trabalho e o papel dos Polos de Capacitação e todo o movimento envolvendo Cosems e gestores municipais, universidades e entidades da comunidade.

Palavras-chave: Atenção Primária à Saúde; Programa Saúde da Família; Formulação de Políticas; Acesso. 


\section{Abstract}

This article describes the development of Programa Saúde da Família (PSF - Family Health Program) in the State of São Paulo since its beginning until the year 2002. The background goes back to the mid 70s, when the Ministry of Health initiated a program to establish health care in distant undeveloped rural areas of the country. Other important antecedents were a similar program conducted by the government of the State of São Paulo in "Vale do Ribeira" from 1980 to 1987 , and the National Program of Community Health Agents, carried out in the 1990s. The article also points out the influence of the São Paulo Metropolitan Region's Health Assistance Program conducted by the State Government and the engagement of activist social health organizations in the city of São Paulo. The description highlights the main characteristics of the PSF in the State of São Paulo and the team's composition in different periods of time. The Government of São Paulo used three different forms to support the development of the program: the first was technical advice and coordination, the second, technical and financial support to the municipalities, and the third was the partnership with non-profit health organizations to establish family health teams in the city of São Paulo. An evaluation of all the family health teams in the State of São Paulo, carried out in 2001, revealed great satisfaction of the assisted population and also examined different forms to engage or hire health professionals to work in family health teams. The development of the program is a consequence of the efforts to promote the qualification of the family health professionals, as well as the engagement of municipalities - especially those responsible for $\mathrm{Pu}$ blic Health Administration, Universities and social health activism.

Keywords: Primary Health Care; Family Health Program; Policymaking; Access.

\section{Antecedentes}

A implantação do Programa de Saúde da Família (PSF), hoje designado Estratégia Saúde da Família (ESF), começou em 1994 no nordeste do Brasil, nas cidades de Sobral e Quixadá, no Ceará, e foi precedido pelo Programa Nacional de Agentes Comunitários de Saúde (PACS), em 1991, cuja experiência trouxe os agentes comunitários de saúde (ACS) para compor as equipes de saúde da família, provocando mudança significativa no processo de trabalho em saúde.

Os ACS eram escolhidos na própria comunidade onde viviam, deles se exigindo algum grau de liderança e apenas saber ler e escrever. A figura do Agente de Saúde do Programa de Interiorizações de Ações de Saúde e Saneamento (PIASS), que foi realizado inicialmente no nordeste, entre 1976 e 1979, expandindo-se para outras regiões do país, posteriormente, deu lugar ao ACS. O PIASS também foi implantado na região do Vale do Ribeira, no Estado de São Paulo, no período de 1980 a 1987 , ficando conhecido como Projeto Devale.

Em 1987 o PACS teve início Ceará, em princípio como frente de trabalho "em situação crítica de seca". O sucesso provocado pelo trabalho realizado casa a casa pelos ACS fez com que, em 1991, o Ministério da Saúde desse início ao Plano Nacional de Agentes Comunitários de Saúde, obtendo impactos importantes na redução da mortalidade infantil, aumento do aleitamento materno exclusivo, aumento da cobertura vacinal em crianças e gestantes e queda das doenças infectocontagiosas, de maneira geral.

Ainda na década de 1980, a região metropolitana de São Paulo foi objeto de um projeto de reestruturação da rede de prestação de serviços de saúde - o Programa Metropolitano de Saúde (PMS) - proposto pela Secretaria Estadual da Saúde (SES), que envolvia prefeituras de vários municípios da região.

Essa região do Estado de São Paulo foi considerada a mais carente de serviços de saúde e de outros serviços públicos, em levantamentos realizados por técnicos da SES-SP e das Secretarias Municipais de Saúde (SMS), que constataram também que as populações da periferia da Grande São Paulo apresentavam péssimas condições de saúde. A decisão para o lançamento do projeto foi uma resposta a movimentos de moradores, especialmente da zona 
leste da cidade de São Paulo, que, agrupados em Comissões de Saúde nos bairros e regiões, exigiam uma ação mais efetiva do governo estadual visando à construção de novos equipamentos de saúde e a melhoria do atendimento assistencial. Os resultados desses levantamentos, aliados a conclusões de estudos anteriores, realizados por médicos sanitaristas e técnicos da SES, possibilitaram um detalhado diagnóstico das condições de saúde e, em especial, da oferta de serviços de saúde na região da Grande São Paulo e já indicavam a necessidade de um tratamento cuidadoso da situação de saúde da metrópole paulista, em especial nos bairros mais distantes.

O modelo de atenção proposto foi o do módulo de saúde, que previa a integração da rede básica a um hospital geral, sob um mesmo comando.

A grande característica desse modelo foi a sua concepção como rede de serviços, ambulatoriais e de internação, organizados sistêmica e hierarquicamente em níveis de complexidade, articulados funcionalmente sob gerência única. A Unidade Básica de Saúde (UBS) seria a porta de entrada da clientela ao sistema, que prestaria assistência integral à saúde de uma área geográfico-populacional delimitada.

Esse programa apontou áreas prioritárias para sua implantação, dentre elas os bairros de Freguesia do Ó e Itaquera/Guaianazes, em São Paulo.

Nesse mesmo período já se desenvolvia na zona leste de São Paulo uma experiência conduzida pelas irmãs Marcelinas de construção de um pequeno consultório médico, com ajuda da própria população. Foi uma primeira iniciativa de tentar mobilizar alguns profissionais para a atenção primária. Seguiu-se uma segunda experiência, adotada em parceria da SES-SP com o Hospital Santa Marcelina, de 1989 a 1995, que foram os consultórios médicos de família, inspirados no modelo cubano.

\section{O PSF no Estado de São Paulo}

A partir de 1996, a SES-SP fez um investimento grande para realizar a mudança do modelo de assistência à saúde da população de São Paulo, tentando garantir assistência integral aos indivíduos, às famílias e à comunidade. Com esse objetivo, o Estado de São Paulo investiu e apostou na implantação, em todo o
Estado, do Programa Saúde da Família.

Com a proposta do PSF, atual Estratégia Saúde da Família, apresentava-se a oportunidade de se viabilizar uma nova forma de atenção à saúde das pessoas, para isso era fundamental assumir o compromisso de propiciar condições para transformar as práticas de saúde que fragmentam as pessoas, os saberes e os serviços de saúde, separando-os da realidade de vida e, portanto, desumanizando a atenção, além de dificultar, pela maneira como essas práticas eram organizadas, uma prestação de assistência integral.

A estratégia de Saúde da Família concretiza-se a partir do trabalho da equipe de Saúde da Família (ESF), cuja equipe mínima deve ser constituída por 1 médico, 1 enfermeiro, 1 ou 2 auxiliares de enfermagem e de 4 a 6 ACS, que assumem a responsabilidade por uma área geográfica definida e sua população e desenvolvem ações de saúde dos indivíduos e da família, dos recém-nascidos aos idosos, sadios ou doentes, de forma integral e contínua.

A atenção está centrada na família, entendida e percebida a partir do seu ambiente físico e social, o que possibilita uma compreensão ampliada do processo saúde/doença e o levantamento de seus problemas de saúde mais expressivos, buscando-se soluções, seja na rede de serviços de saúde seja na sociedade em geral, através da intersetorialidade. Trata-se de um atendimento humanizado, resolutivo e de qualidade.

A decisão do Governo Estadual de investir na reorientação do modelo da assistência no sentido da assistência integral e, nesse processo, de apoiar a implantação do PSF em todo o Estado, orientou o desencadear de três importantes movimentos, nessa direção.

\section{Apoio Técnico aos Municípios para que Implantassem o Programa}

A implantação do PSF nos municípios do Estado deveu-se, em parte, a um grande movimento pela sensibilização dos gestores municipais e das instituições de ensino.

Para tanto, a SES-SP propiciou intenso debate entre gestores municipais, profissionais de saúde, instituições de ensino e sociedade. A parceria 
com o Cosems-SP foi fundamental nas atividades destinadas à sensibilização para as "vantagens" da estratégia, debatendo seus princípios e diretrizes em discussões relacionadas às formas de financiamento ou ainda discutindo qual a melhor forma de contratar profissionais para atuar na estratégia e como fazer isso. Questões iam se apresentando quanto mais se caminhava na sua implantação, uma vez que no inicio não havia experiências ou práticas suficientes sobre o Programa.

No Estado de São Paulo, o PSF teve início em 1996 com a implantação de Equipes de Saúde da Família em alguns municípios. Em 2002 o programa já contava com 1.638 equipes de PSF implantadas em 402 municípios, possibilitando uma cobertura de cerca de $20 \%$ da população do Estado, isto é, mais de 5,6 milhões de pessoas.

Entre os municípios do Estado de São Paulo, houve uma grande aceitação do PSF, o que se refletiu no número de municípios que decidiram implantar o programa. Pode-se observar, no gráfico a seguir, uma tendência de redução do número de municípios com PACS e o crescimento daqueles que introduziram o PSF, ao longo do período, refletindo o interesse pelo modelo mais completo e resolutivo, como seria de se esperar num estado com boa rede de atenção básica.
Gráfico I - Número de equipes de saúde da família, por ano, no estado de São Paulo, de 1998 a 2002

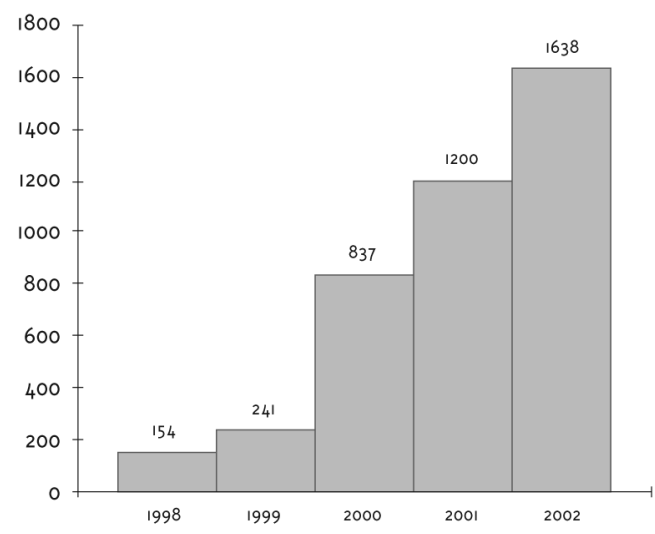

Gráfico 2 - População coberta pelas equipes de saúde da família, por ano, no estado de São Paulo, de 1998 a 2002

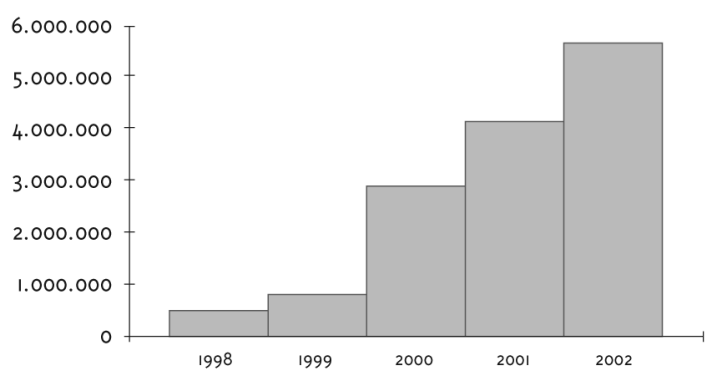

Fonte: Secretaria de Estado da Saúde de São Paulo. Dados de 2002 apenas até o mês de agosto.

\section{Grafico 3 - Relação entre implantação do Programa Saúde da Família (PSF) e do Programa de Agentes Comuni- tários de Saúde (PACS) nos municípios paulistas no período de julho de 1998 a julho de 2002}

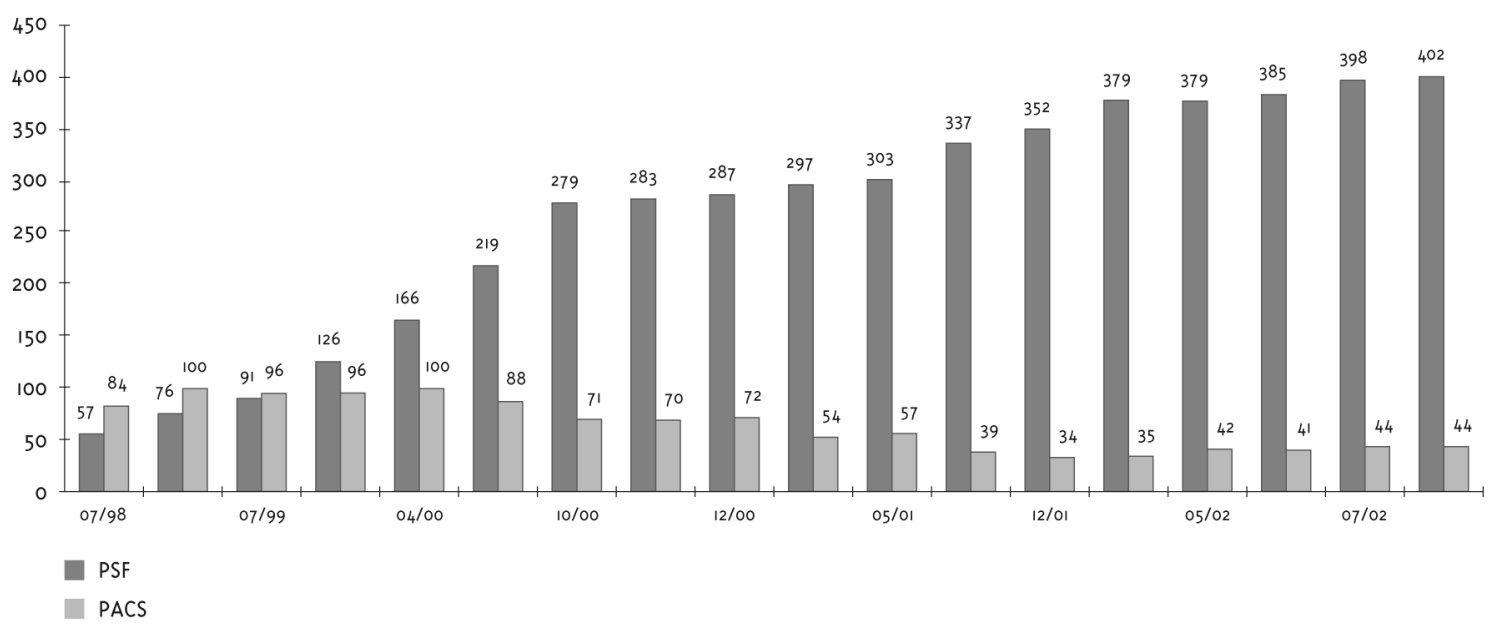




\section{Em Municipios Prioritários do Interior do Estado}

Em 1999, a SES-SP decidiu financiar e garantir a implantação da ESF no interior do Estado, contemplando regiões consideradas prioritárias, tendo em vista as péssimas condições de saúde e as dificuldades de acesso da população. Foram considerados os seguintes critérios:

- baixo índice de Desenvolvimento Humano (IDH) na Região Vale do Paraíba e Litoral Norte, Vale do Ribeira e Região Itapeva/Itararé, Região Oeste, Unipontal;

- áreas de assentamento, em todo o Estado;

- comunidades remanescentes de Quilombos.

Nesses municípios, o Estado foi responsável pelo apoio financeiro que representava 2/3 do custeio das equipes, além de apoio técnico.

Às áreas de assentamento rural e comunidades remanescentes de Quilombos, devido a características geográficas e dificuldade de acesso das populações, foram destinados recursos de investimentos em comunicação (como, rádio amador, telefone celular rural), meio de transportes (bicicletas, carroças, motocicletas, barcos e carros), adequação das unidades para o atendimento (construção ou reformas) e aquisição de equipamentos, que foram escolhidos pelos representantes das famílias assentadas ou quilombolas, conforme as necessidades das populações da área.

Até agosto de 2002, o Qualis/PSF dos municípios prioritários do interior tinha sido implantado em 117 municípios, cobrindo cerca de 1 milhão de pessoas com o trabalho de 269 equipes de saúde da família. O estado de São Paulo aplicou, apenas em 2001, recursos no valor de R\$ 26,1 milhões no programa.

\section{Na Capital do Estado}

No município de São Paulo, capital, desenvolveuse a partir de abril de 1996 a primeira experiência nacional do Programa de Saúde da Família, em grandes cidades, o Projeto Qualidade Integral em Saúde (Qualis). O prefeito da época optou pelo Plano de Assistência à Saúde (PAS), cujo modelo reforçava as limitações da visão biomédica, colaborando com parcos resultados para a melhoria da saúde da população.

O Ministério da Saúde, a Secretaria Estadual da Saúde e a entidade filantrópica Casa de Saúde Santa Marcelina (CSSM) estabeleceram parceria para efetivar o PSF no município.

Foi no projeto da capital que a SES-SP esteve mais perto dos parceiros acompanhando e participando de todo o seu desenvolvimento.

A CSSM localiza-se na zona leste do município de São Paulo desde 1961. Dirige um hospital com mais de 700 leitos, referência para toda a região. Possui Centro de Ensino e Pesquisa, que promove a formação de especialistas, ofertando diversos programas de residência médica, internato médico, além de manter Postos de Saúde próximos ao hospital, que há vários anos desenvolvem projetos em atenção primária.

A experiência adquirida pela Casa de Saúde Santa Marcelina e sua estrutura assistencial permitiria desenvolver um Programa de Saúde da Família que, por contar com ambulatórios de especialidades e um centro hospitalar, garantiria a integralidade da assistência, que foi a marca que a SES-SP fez questão de dar ao programa.

Foi no projeto Qualis I, com o Santa Marcelina, que as equipes do hospital e da SES-SP aprenderam, no trabalho diário, a construir o novo modelo.

A experiência dos técnicos em educação da SESSP trouxe grandes ensinamentos aos profissionais do Qualis, incentivando a discussão do processo de trabalho nas UBS. Como o número de equipes crescia continuamente, aumentando a demanda por educação, criou-se o Núcleo de Educação Permanente, em 1997, estabelecendo o trabalho como eixo fundamental para a aprendizagem.

A defesa do trabalho em equipe retirava do médico a centralidade das decisões. A produção do cuidado envolvia todos os profissionais e diferentes saberes, contemplando inclusive a sabedoria popular, trazida pelos ACS e pela comunidade.

Ao iniciar o trabalho na comunidade, a equipe Qualis fazia o reconhecimento do território, dividindo-o em microáreas, de acordo com o número de ACS que, obrigatoriamente, deveriam morar em cada uma das microáreas. Nessa ação, a equipe entrava em contato com as lideranças locais, que 
eram chamadas para reunião realizadas geralmente nos salões paroquiais ou sede de alguma associação de bairro. Mapeava-se o território e identificavamse áreas de risco, como lixões, favelas, córregos, e também outros equipamentos sociais, como escolas, creches, etc.

Na etapa seguinte fazia-se o diagnóstico situacional, unindo dados oficiais aos da pesquisa direta feita de casa em casa, com aplicação do cadastro familiar, a ficha A do Sistema de Informação da Atenção Básica (SIAB). A partir daí a equipe se reunia, microárea por microárea, construindo o planejamento das ações de saúde.

O ACS realizava as visitas domiciliares, atualizava os cadastros mensalmente e trazia para o encontro diário com a enfermeira as demandas da população. As necessidades de saúde da população se explicitavam, exigindo novas ações e desafiando as potencialidades do serviço assistencial, que exigiam a incorporação de outros profissionais à equipe mínima do PSF.

O cadastramento das famílias permitiu conhecer a realidade de cada um dos usuários dos territórios incorporados ao projeto, absorvendo a complexidade das demandas nem sempre rotuladas como "doenças". O desemprego e a violência foram os grandes desafios para as equipes.

Ficou evidente a necessidade de se trabalhar a intersetorialidade de forma mais abrangente, envolvendo outras Secretarias Municipais e Estaduais do Governo, além de ONGs e outros organismos da sociedade civil. Duas Casas de Parto foram criadas, a uma delas em Sapopemba, por iniciativa do Dr. David Capistrano, e a outra na zona leste, ligada ao Hospital do Itaim Paulista, dirigida pelas Irmãs Marcelinas. As gestantes podiam ser atendidas próximo de suas residências, assistidas por obstetrizes, acompanhadas por membros da família, humanizando-se o serviço.

Foi possível o acompanhamento das puérperas e dos bebês, articulando-se o pré-natal, o parto e o puerpério.

Foram criadas 31 Cooperativas de Trabalho, na zona leste, gerando renda para os desempregados, em parceria do Qualis com a Secretaria Estadual do Emprego e Trabalho.

A Secretaria Municipal do Meio Ambiente aju- dava a desenvolver hortas comunitárias, educação para preservar o meio ambiente, etc. A coleta de lixo foi adaptada para a realidade de áreas de invasão, nas quais as ruas com traçado impreciso e muito estreitas dificultavam a chegada do caminhão de lixo. Lixeiras coletivas foram construídas, ruas foram cascalhadas, construíram-se creches. A atuação dos ACS junto à população foi fundamental para se efetivar as conquistas.

O contato direto dos trabalhadores da saúde com a comunidade gerou muito sofrimento. Era difícil conviver com problemas para os quais não se tinha resposta imediata. Só a mobilização da comunidade, a verdadeira interessada nas transformações sociais, poderia modificar a realidade. Era comum o desemprego, a violência de gênero, o espancamento de crianças, a precariedade das habitações, o analfabetismo, a miséria. A SES-SP escolheu trabalhar essas áreas que eram as mais carentes.

A falta de preparo da equipe de saúde para lidar com esses problemas exigia discussões contínuas entre os seus próprios componentes e entre eles e a comunidade, utilizando-se o apoio do Núcleo de Educação Permanente, formado por técnicos da SES.

O contato direto com o território, como ele se organiza, qual a sua dinâmica e articulação com os diversos grupos de indivíduos, famílias e comunidade, ensinou a equipe de saúde e o povo a entender o processo saúde-doença, a trabalhar seus determinantes, a buscar a intersetorialidade para dar conta dos problemas.

A experiência do Projeto Qualis, mobilizadora de tantos outros projetos, mostrou que é possível construir caminhos que coloquem o usuário na centralidade da atenção em saúde. Foi para cuidar da saúde das pessoas que se idealizou um sistema tão complexo, tão moderno e tão inclusivo como é o sistema de saúde brasileiro.

Após essa experiência inicial, a articulação da SES-SP em parceria com a Fundação Zerbini viabilizou a implantação do Projeto Qualis II a partir de novembro de 1997, nas zonas sudeste (Parque São Lucas e Sapopemba) e norte (Vila Nova Cachoeirinha) da cidade. Em 1998, a SES-SP inaugurou dois ambulatórios de especialidades (cardiologia, oftalmologia, otorrinolaringologia, pneumologia, endocrinologia, neurologia, psiquiatria, urologia, ginecologia, gas- 
troenterologia e medicina do trabalho), integrados às UBS: um na unidade do Jardim Guaraicá (Parque São Lucas/Sapopemba) e o outro na unidade de Vila Espanhola (na Vila Nova Cachoeirinha). Teve início então a atuação de equipes de saúde bucal integradas por odontólogos, técnicos de higiene dental e atendentes de consultório dentário.

O êxito da iniciativa da SES-SP com a CSSM e a Fundação Zerbini orientou, em 2000, a parceria com a Universidade Santo Amaro (Unisa) e a Associação Congregação Santa Catarina, expandindo o programa para a região sul da capital. Essas novas parcerias se beneficiaram do conhecimento e da experiência adquiridos no desenvolvimento do Programa da zona leste, norte e sudeste. 0 modelo assistencial se repetiu: a Congregação Santa Catarina assumiu o Hospital de Pedreira, o Ambulatório de Especialidades Jardim dos Prados e a rede básica, a Unisa assumiu o Hospital do Grajaú, o Ambulatório de Especialidades de Interlagos e a rede de unidades básicas. Nesse mesmo ano, a Direção Regional de Saúde da Grande São Paulo resolveu implantar o Qualis/PSF em unidades próprias da SES.

Das 15 equipes de saúde da família que cobriam cerca de 51.750 pessoas em 1996, o Qualis atingiu 218 equipes, cobrindo 872.000 pessoas, em meados de 2002, quando foi então foi municipalizado. No final de 1998, o Qualis Zerbini foi premiado com o $1^{\circ}$ lugar no Concurso Unibanco - Saúde - Medicina.

Em junho de 2002, todas as unidades básicas do Qualis/PSF do município de São Paulo e suas 218 equipes de saúde da família já haviam passado para a responsabilidade da Secretaria Municipal de Saúde.

\section{Recursos Humanos}

Uma vez decidida a implantação do PSF, a capacitação das equipes e dos gestores tornou-se um desafio. Todos os membros das equipes implantadas passavam por um treinamento introdutório para conhecer o novo modelo de atenção e o processo de trabalho. Esse treinamento era aplicado pelos interlocutores do PSF nas Regionais de Saúde da SES-SP.

Para viabilizar a implantação do PSF e garantir sua qualidade e continuidade, o Ministério da Saúde desenvolveu a estratégia de Polos de Capacitação, espaço de articulação de universidades e outras instituições de ensino. Em São Paulo, tendo em vista a sua complexidade e dimensão, foram organizados cinco polos regionais de capacitação - Polo da Região Metropolitana da Grande São Paulo, Polo de Marília, Polo de Botucatu, Polo de Ribeirão Preto/São José do Rio Preto e Polo de Campinas - articulados no Sistema Estadual de Capacitação, Formação e Educação Permanente de Recursos Humanos para a Saúde da Família.

Tratava-se e uma rede de instituições comprometidas com a integração Ensino/Serviço, voltada para atender à demanda de pessoal preparado para o desenvolvimento da estratégia de saúde da família, no âmbito do SUS de São Paulo. Esses polos desenvolveram e garantiram treinamentos nas áreas de atenção básica para médicos, enfermeiros, auxiliares de enfermagem e agentes comunitários de saúde.

Somente no ano de 2002, os Polos de Capacitação de RH em Saúde da Família da Grande São Paulo e Santos, de Marília e o Núcleo Norte do Polo NorteOeste Paulista capacitaram 7.094 profissionais: 554 médicos, 891 enfermeiros, 858 auxiliares de enfermagem, 3.871 agentes comunitários de saúde, 288 cirurgiões-dentistas, 64 atendentes de consultório dentário e 568 profissionais de outras categorias ligadas à área da saúde.

Esses profissionais receberam treinamentos em Módulo Introdutório e Saúde da Família, Saúde da Mulher, Saúde da Criança, Diabetes, MH, TBC, Saúde Bucal, Dengue, DST/Aids e SIAB.

Os Polos de Capacitação, através de 14 Cursos de Especialização Multiprofissional em Saúde da Família, titularam 410 profissionais, além de oferecer Residência Multiprofissional em Saúde da Família.

No Polo de Capacitação da Grande São Paulo e Santos a Universidade Federal de São Paulo e a Faculdade de Medicina da Santa Casa de São Paulo, juntas, concluíram 3 Cursos de Especialização em Saúde da Família.

\section{O Movimento pela Capacitação}

Pode-se dizer que nos primeiros anos de implantação do PSF no Estado de São Paulo ocorreu uma 
verdadeira "peregrinação" para a viabilização do programa, com a promoção de vários encontros e eventos, realizados em quase todas as regionais de saúde do Estado e que superaram sempre a expectativa de participação.

O I Encontro dos Polos de Capacitação em PSF, realizado no final de 1998 na cidade de Atibaia, que contou com a participação de representantes de todas as escolas médicas e de enfermagem envolvidas nos polos, representantes da SES-SP, Cosems, Coren, CRM, MS e teve a participação de mais de cem profissionais.

O Encontro dos cinco Polos de Capacitação do Sistema Estadual de Capacitação em Saúde da Família, em 1999, na cidade de São Pedro, interior de São Paulo, em que quase 200 profissionais puderam aprofundar e trocar experiências sobre a formação e qualificação de profissionais.

A I Mostra de Saúde da Família do Estado de São Paulo, que aconteceu, em 200o, no município de Ribeirão Preto, promovida pela SES-SP em parceria com o Cosems e com o apoio do Ministério da Saúde e da Fundação do Remédio Popular (FURP), na qual estiveram presentes mais de 2.000 participantes, entre eles representantes de cerca de 250 municípios, as universidades que compunham o Sistema Estadual de Educação Permanente, profissionais de saúde e membros da comunidade.

Se por um lado, a expansão acelerada do PSF no Estado trouxe para a SES-SP e as Secretarias Municipais de Saúde muitos desafios, por outro, acumulou informações na busca de soluções criativas que precisavam ser partilhadas e debatidas.

Os resultados quantitativos e qualitativos do programa, apropriados pelas equipes, agentes comunitários, gestores e a própria comunidade suscitaram discussões calorosas sobre o melhor gerenciamento das realidades locais de saúde. Além disso, questões mais amplas, como o desafio de gestão, a contratação e capacitação de pessoal, o planejamento, o processo de trabalho, a administração de recursos, a articulação com os demais níveis do sistema, foram temas de comunicações coordenadas, pôsteres interativos e várias mesas-redondas.
O sucesso da I Mostra Estadual de PSF superou as expectativas, demonstrando a vontade e a decisão política dos gestores, a competência técnica dos profissionais envolvidos na promoção da saúde e na conquista da cidadania, demonstrando que é possível mudar a abordagem da atenção à saúde com resultados positivos, independentemente das dimensões geográficas, econômicas ou políticas dos nossos municípios.

O município de Assis foi palco, em 2001, do I Encontro de Agentes Comunitários de Saúde, que teve a participação de mais de 1.300 agentes que trabalhavam em mais de 200 municípios do Estado de São Paulo. Com o objetivo de promover intercâmbios e reflexões sobre o papel do ACS no contexto do SUS, o esse encontro também promoveu a fundação da Federação Paulista de Agentes Comunitários de Saúde. 0 grande triunfo desse encontro "Semeando novas Sementes" foi a criação das organizações de agentes comunitários de saúde nos municípios e a integração desse novo ator no SUS, seu papel no avanço e na renovação das ações na promoção de saúde e cidadania. Os ACS no Estado não contavam até então com nenhuma entidade que os representasse.

Além dos grandes eventos estaduais, vários encontros regionais foram realizados pelos Conselhos Intergestores Regionais de Saúde, com a participação de gestores estaduais e municipais da região, das instituições de ensino envolvidas, dos profissionais das equipes e muitas vezes de prefeitos municipais, que relatavam experiências positivas e também as dificuldades encontradas para a implantação do PSF.

\section{Avaliação}

Uma pesquisa avaliativa da implantação do PSF foi realizada pelo Ministério da Saúde, em parceria com as Coordenações Estaduais do PACS/PSF em todos os estados da Federação'.

No Estado de São Paulo, além da pesquisa avaliativa das equipes de saúde da família, o processo de avaliação envolveu os gestores - buscando conhecer

\footnotetext{
1 Ministério da Saúde. Avaliação da Implantação do Programa de Saúde da Família/Programa de Agentes Comunitários de Saúde - Relatório de Pesquisa. Brasília: Ministério da Saúde, 2001 (mimeo).
} 
as razões pelas quais foi implantado o PSF e como eles o avaliavam - e a comunidade - buscando conhecer a utilização dos serviços e aceitação do Programa pela população cadastrada.

Foram entrevistadas 1.058 equipes (ou seja, $100 \%$ das equipes implantadas até setembro de 2001), 2.077 famílias cadastradas escolhidas aleatoriamente (duas famílias por equipe) e 310 gestores de saúde de municípios que haviam implantado o programa.

Os resultados da pesquisa de avaliação das equipes foram apresentados e discutidos com o Ministério da Saúde, as DIRs, os gestores, os coordenadores de PSF e os polos de capacitação, com o objetivo de melhorar a qualidade da atenção e readequar a capacitação dos profissionais pelos polos.

A pesquisa de avaliação da população mostrou boa aceitação do programa pela população, 8o,4\% das famílias entrevistadas consideraram o PSF bom ou ótimo; $14,4 \%$ regular e $5,4 \%$ ruim ou péssimo. Em $57 \%$ dos municípios todas as famílias entrevistadas avaliaram o atendimento como bom ou ótimo, sugerindo que, se bem implementado, o programa teria boa aceitação pelos usuários.

Outro aspecto importante que a pesquisa demonstrou foi que no Estado de São Paulo a precarização do trabalhador das equipes, incluindo os ACS, era muito pequena, isto é, a maioria dos trabalhadores (cerca de 70\%) apresentava vínculos empregatícios -, o número de funcionários públicos ou sob regime de trabalho pela CLT, contratos de trabalho temporários foi de $15 \%$ a 20\%, funcionários sem nenhum contrato $5 \%$.

\section{Considerações Finais}

Nestes primeiros sete anos de implantação do PSF no Estado de São Paulo, houve uma importante participação da SES, desenvolvida em parceria, principalmente com o Cosems e com as Instituições de Ensino integrantes dos Polos de Capacitação em Saúde da Família.

Durante esse período, foram levantadas, debatidas e encaminhadas inúmeras questões relativas à implantação das equipes de saúde da família nos municípios.

Pode-se dizer que foram anos de intenso conhecimento do "novo", buscando-se a forma de "como fazer”, um verdadeiro desafio, principalmente levando em consideração mudanças que deveriam ser realizadas em um Estado com grande capacidade tecnológica e com práticas de saúde já consolidadas em seus vários municípios, além da sua dimensão populacional.

Assim, o protagonismo de implantar o PSF na periferia do município de São Paulo e destinar recursos próprios para o custeio para equipes de saúde da família em municípios de baixo IDH, com população assentada e/ou quilombola fez parte dessa caminhada. Entretanto o caminho ainda é longo e algumas questões permanecem, como: Qual o percentual dos recursos de saúde deve ser aplicado para uma APS adequada, que possa efetivamente exercer seu papel de coordenadora do cuidado nos sistemas de saúde de forma integral e resolutiva? Como atrair, alocar e fixar recursos humanos na ESF, notadamente o médico generalista e/ou de família? Como qualificar esses profissionais para que daqui a dez anos todos os médicos da ESF tenham residência em Medicina de Família e Comunidade?

Para que o PSF seja implantado é necessário que sejam providas condições adequadas para seu efetivo processo de trabalho, isto é, que haja adequada estrutura física das unidades de saúde, fornecimento de insumos e equipamentos médicos e de enfermagem, além do estabelecimento de pactos com os profissionais de saúde, baseados na construção de vínculos com o usuário, com a comunidade, com a responsabilidade de se obter resultados satisfatórios de suas intervenções e compromissos com a melhoria de saúde das pessoas.

Para finalizar, é importante destacar o papel fundamental para o alcance desses resultados: o incentivo de Adib Jatene, no Ministério da Saúde, o entusiasmo de Mário Covas, no Governo do Estado, a articulação e a coordenação de ações inovadoras da Secretaria de Estado da Saúde através de seus profissionais, o grande empenho dos municípios, a dedicação e a competência das equipes dos parceiros filantrópicos e a pressão e a participação da população.

Recebido em: 26/05/20II

Aprovado em: 15/07/2011 